\title{
2020 : année la plus chaude en France, I'une des trois les plus chaudes dans le monde
}

En France, l'année 2020 est l'année la plus chaude de la période 19002020 (figure 1), avec une température moyenne de $14,07{ }^{\circ} \mathrm{C}\left(1,52{ }^{\circ} \mathrm{C}\right.$ au-dessus de la normale 1981-2010 et $1,13{ }^{\circ} \mathrm{C}$ au-dessus de la future normale 1991-2020), devant $2018\left(+1,39{ }^{\circ} \mathrm{C}\right)$ et $2014\left(+1,2^{\circ} \mathrm{C}\right)$. Sauf octobre (déficit de $\left.0,5{ }^{\circ} \mathrm{C}\right)$, tous les mois ont une moyenne excédentaire (tableau 1); c'est en février et en avril que l'excédent est le plus fort (respectivement $+3,6{ }^{\circ} \mathrm{C}$ et $+3,0{ }^{\circ} \mathrm{C}$ ). L'anomalie positive est plus marquée dans le Nord et le Nord-Est que dans les régions méditerranéennes; elle est également plus marquée sur les températures maximales $\left(+1,92^{\circ} \mathrm{C}\right)$ que sur les minimales $\left(+1,12{ }^{\circ} \mathrm{C}\right)$. Les dix années les plus chaudes depuis 1900 se situent au XXI ${ }^{\mathrm{e}}$ siècle, à l'exception de 1994. La décennie 2011-2020 est la plus chaude depuis au moins 1900 ; avec une moyenne de $13,44{ }^{\circ} \mathrm{C}$, elle devance 2001-2010 (12,84 $\left.{ }^{\circ} \mathrm{C}\right)$. Quant à la période trentenaire 1991-2020, elle a une moyenne de $12,94{ }^{\circ} \mathrm{C}$ contre des moyennes de $12,53{ }^{\circ} \mathrm{C}$ pour $1981-2010$ et $11,81^{\circ} \mathrm{C}$ pour $1961-1990$.

La France a connu plusieurs vagues de chaleur : du 20 au 23 mai; du 26 juillet au $1^{\text {er }}$ août $\left(41,9^{\circ} \mathrm{C}\right.$ à Socoa (Pyrénées-Atlantiques), $41^{\circ} \mathrm{C}$ à Grospierres (Ardèche) et $20,1^{\circ} \mathrm{C}$ au pic du Midi, égalant le record du 20 juillet $1995)$; du 6 au 13 août $\left(41,9^{\circ} \mathrm{C}\right.$ à Belis (Landes), $41,2^{\circ} \mathrm{C}$ à Orange (Vaucluse),

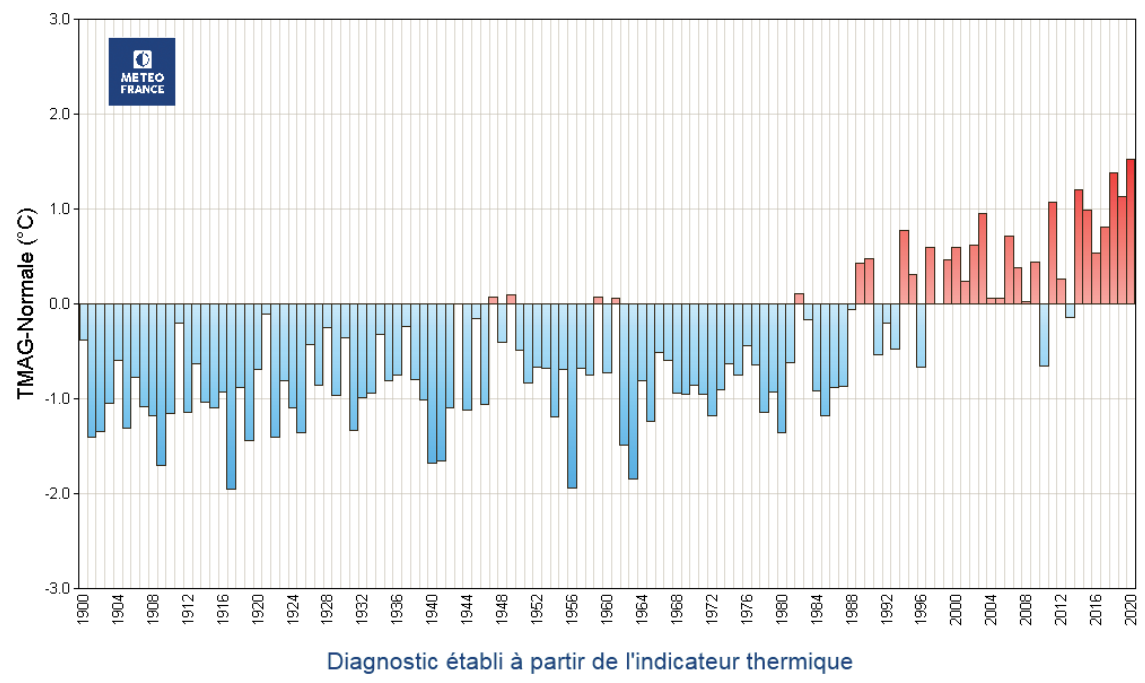

Figure 1. Écart à la normale 1981-2010 des températures moyennes de 1900 à 2020 en France. Source : https://www.meteofrance.fr/climat-passe-et-futur/bilans-climatiques/843/bilan-climatiquede-I-annee-2020

$40,9{ }^{\circ} \mathrm{C}$ à Gourdon (Lot)) ; du 13 au 17 septembre $\left(37,8{ }^{\circ} \mathrm{C}\right.$ à Dax (Landes) ; le 14, la température maximale moyenne en France a atteint $33,4{ }^{\circ} \mathrm{C}, 11{ }^{\circ} \mathrm{C}$ au-dessus de la normale !). Parmi les autres événements marquants de l'année, citons les nombreuses tempêtes de janvier à mars et les épisodes méditerranéens : du 21 au 24 janvier sur l'Aude et les Pyrénées-Orientales (précipitations maximales de $427 \mathrm{~mm}$ à Arles-surTech) ; les 11 et 12 juin sur les Cévennes (456 mm à Vialas); les 18 et 19 septembre sur les Cévennes $(500 \mathrm{~mm}$ à
Valleraugue) et surtout le 2 octobre sur les Alpes-Maritimes où les vallées de la Roya, de la Vésubie et de la Tinée subissent une terrible catastrophe (513 mm à Saint-Martin-Vésubie ; dégâts considérables et neuf morts). En décembre, le Sud-ouest connaît pluies diluviennes et inondations.

$\mathrm{Au}$ niveau mondial, plusieurs organismes analysent l'évolution de la température. Leurs résultats présentent de légères différences, mais montrent que trois années (2020, 2019 et 2016) ont des températures annuelles très

Tableau 1. Écarts mensuels des températures $\left({ }^{\circ} \mathrm{C}\right)$ de 2020 dans le monde par rapport aux normales 1901-2000 et rang depuis 1880. Températures en France et écarts par rapport à la normale 1981-2010. Sources : NCDC/Noaa et Météo-France.

\begin{tabular}{lcccccccccccc} 
& \multicolumn{2}{c}{ Monde } & \multicolumn{2}{c}{ Continents } & \multicolumn{2}{c}{ Océans } & \multicolumn{2}{c}{ Hémisphère Nord } & \multicolumn{2}{c}{ Hémisphère Sud } & France \\
& Écart & Rang & Écart & Rang & Écart & Rang & Écart & Rang & Écart & Rang & Temp. ${ }^{\circ} \mathrm{C}$ & Écart \\
\hline Janvier & $+1,14$ & 1 & $+2,12$ & 1 & $+0,78$ & 2 & $+1,50$ & 1 & $+0,78$ & 2 & 7,1 & $+2,2$ \\
\hline Février & $+1,17$ & 2 & $+2,27$ & 2 & $+0,76$ & 2 & $+1,58$ & 2 & $+0,76$ & 2 & 9,3 & $+3,6$ \\
\hline Mars & $+1,16$ & 2 & $+2,05$ & 2 & $+0,83$ & 2 & $+1,52$ & 2 & $+0,80$ & 3 & 9,4 & $+0,7$ \\
\hline Avril & $+1,06$ & 2 & $+1,65$ & 2 & $+0,83$ & 1 & $+1,28$ & 2 & $+0,84$ & 2 & 14,1 & $+3,0$ \\
\hline Mai & $+0,95$ & 1 & $+1,39$ & 1 & $+0,79$ & 2 & $+1,19$ & 1 & $+0,72$ & 5 & 16,5 & $+1,5$ \\
\hline Juin & $+0,92$ & 3 & $+1,29$ & 3 & $+0,78$ & 3 & $+1,11$ & 3 & $+0,72$ & 3 & 18,6 & $+0,2$ \\
\hline Juillet & $+0,92$ & 2 & $+1,23$ & 2 & $+0,80$ & 3 & $+1,18$ & 1 & $+0,65$ & 7 & 21,6 & $+0,9$ \\
\hline Août & $+0,94$ & 2 & $+1,26$ & 3 & $+0,82$ & 2 & $+1,19$ & 1 & $+0,67$ & 6 & 22,6 & $+2,0$ \\
\hline Septembre & $+0,97$ & 1 & $+1,49$ & 1 & $+0,77$ & 4 & $+1,21$ & 3 & $+0,70$ & 1 & 19,0 & $+1,7$ \\
\hline Octobre & $+0,85$ & 4 & $+1,23$ & 5 & $+0,70$ & 5 & $+1,07$ & 4 & $+0,61$ & 9 & 13,0 & $-0,5$ \\
\hline Novembre & $+0,97$ & 2 & $+1,77$ & 1 & $+0,67$ & 4 & $+1,34$ & 1 & $+0,57$ & 9 & 10,5 & $+2,0$ \\
\hline Décembre & $+0,78$ & 8 & $+1,27$ & 8 & $+0,59$ & 8 & $+1,10$ & 4 & $+0,45$ & 20 & 6,9 & $+1,3$ \\
\hline Année & $+0,98$ & 2 & $+1,59$ & 1 & $+0,76$ & 3 & $+1,28$ & 1 & $+0,68$ & 5 & 14,1 & $+1,5$ \\
\hline
\end{tabular}


proches. Pour le Centre européen pour les prévisions météorologiques à moyen terme (CEPMMT, service Copernicus) et la Nasa, 2020 est en tête (à égalité avec 2016 pour la Nasa) ; la Noaa place 2020 au deuxième rang derrière 2016, avec une différence de $0,02{ }^{\circ} \mathrm{C}$, mais cet organisme ne tient pas compte des régions polaires...; le Met Office britannique met également 2020 en deuxième place, alors que le Service météo japonais la situe au troisième rang... La place de 2020 est d'autant plus remarquable que la fin de l'année a connu le phénomène La Niña qui contribue à abaisser la température moyenne du globe, alors que 2016 avait été une année à El Niño.

En 2020, la température mondiale $\left(14,9^{\circ} \mathrm{C}\right)$ a été supérieure de $1,25^{\circ} \mathrm{C}$ à celle de la période dite préindustrielle (1850-1900) et de $1,04^{\circ} \mathrm{C}$ à la moyenne du $\mathrm{XX}^{\mathrm{e}}$ siècle. Les six années les plus chaudes ont toutes été enregistrées depuis 2015 (figure 2). La figure 3 et le tableau montrent les différences régionales. Quant à la décennie 2011-2020, elle est la plus chaude depuis le début $\mathrm{du} \mathrm{XX}^{\mathrm{e}}$ siècle.

En Europe, selon le Deutsche Wetterdienst (DWD), sur 119 stations de référence, 118 ont eu, en 2020, une moyenne supérieure à la normale 1961-1990. L'année 2020 se situe au premier rang de la période 1880 2020, avec un excédent de $2,16{ }^{\circ} \mathrm{C}$ sur la période 1850-1900. Dans plusieurs pays (France, Suisse, Belgique, Luxembourg, Suède, Norvège, Estonie, Russie), l'année 2020 arrive en tête; elle est deuxième en Allemagne, en Pologne et au Danemark, troisième au Royaume-Uni et cinquième en Italie. En Autriche, 2020 est la cinquième année la plus chaude de la période 1798-2020 dans les vallées, mais est en tête en haute montagne... Au cours de l'été, l'Europe a connu plusieurs vagues de chaleur; les températures les plus élevées ont atteint $45,7{ }^{\circ} \mathrm{C}$

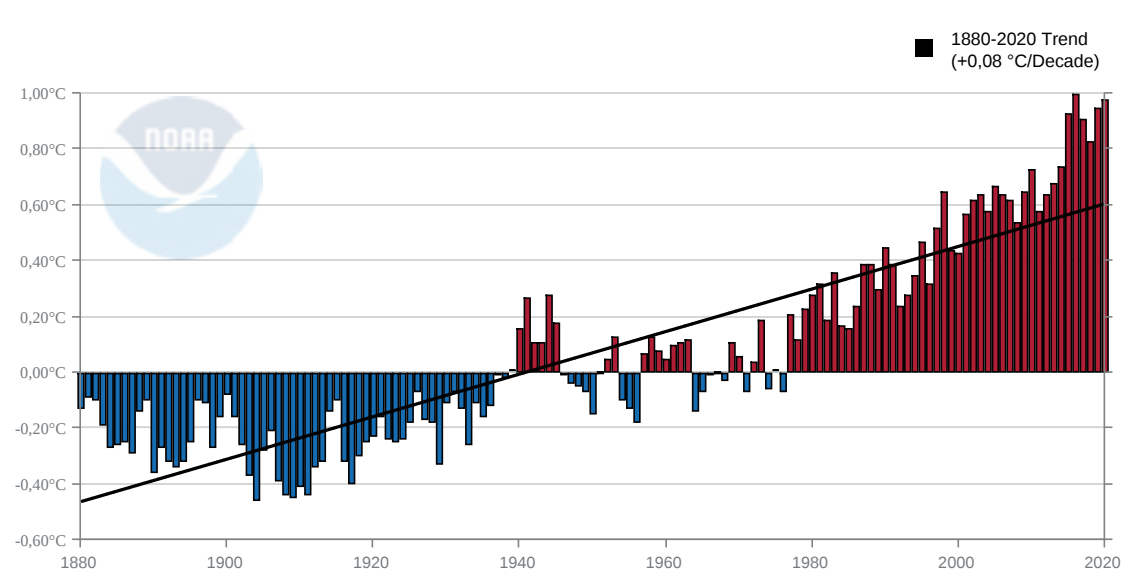

Figure 2. Anomalie de la température mondiale $\left({ }^{\circ} \mathrm{C}\right)$ par rapport à la moyenne du $X X^{e}$ siècle pour la période 1880-2020. Source : NCDC/Noaa. https://www.ncdc.noaa.gov/cag/global/time-series

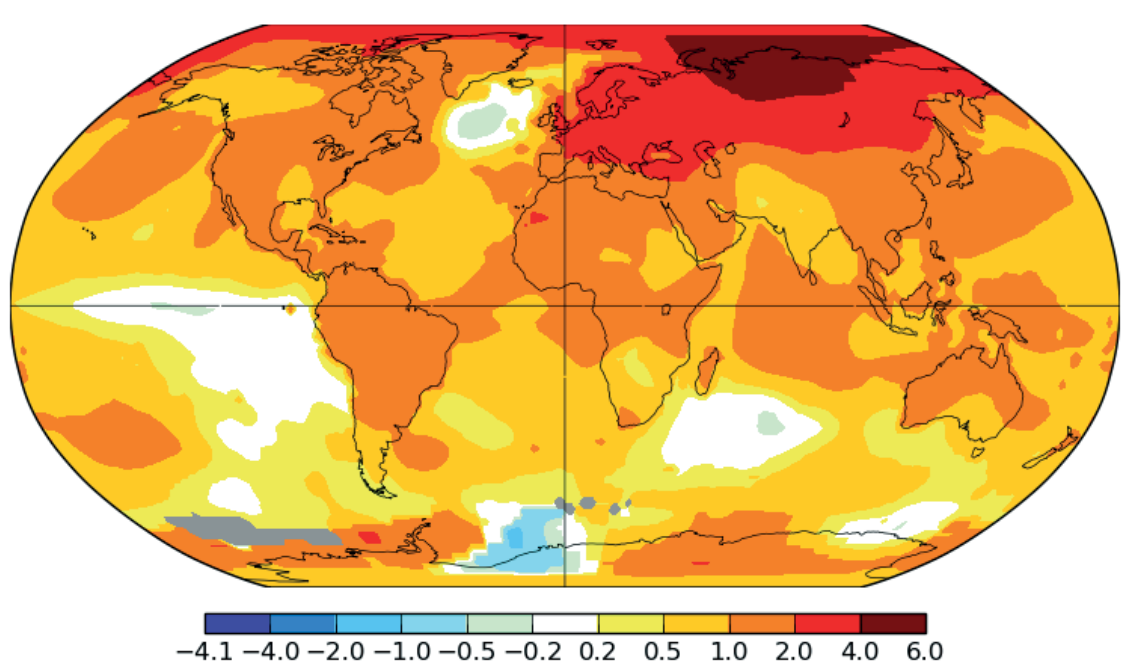

Figure 3. Anomalie de la température $\left({ }^{\circ} \mathrm{C}\right)$ de 2020 par rapport à la moyenne du XXe siècle. Source : Nasa/Giss.

le 2 août à Velez-Malaga (Espagne), $41,3{ }^{\circ} \mathrm{C}$ à Volgograd (Russie), $41{ }^{\circ} \mathrm{C}$ à Mostar (Bosnie-Herzégovine), $40,7^{\circ} \mathrm{C}$ à Podgorica (Monténégro), $40,6{ }^{\circ} \mathrm{C}$ à Palma de Majorque (Espagne), $40,2^{\circ} \mathrm{C}$ à Alméria (Espagne) et $40^{\circ} \mathrm{C}$ à Madrid (Espagne).

En Asie, selon le DWD, 133 stations sur les 136 de référence ont eu une température excédentaire ; 2020 se place au premier rang de la période 1880-2020. Les écarts à la moyenne annuelle les plus importants dépassent $3{ }^{\circ} \mathrm{C}$ en Sibérie $\left(5^{\circ} \mathrm{C}\right.$ à Vitim, $4,7^{\circ} \mathrm{C}$ à Iakoutsk, $3,7^{\circ} \mathrm{C}$ à Omsk et Tobolsk) ; en revanche, ils sont plus modestes au Moyen-Orient $\left(1,9{ }^{\circ} \mathrm{C}\right.$ à Koweït et à Eilat, $1^{\circ} \mathrm{C}$ à Médine, $0,7^{\circ} \mathrm{C}$ à Téhéran) et en Chine $\left(1,9^{\circ} \mathrm{C}\right.$ à Pékin et $0,9^{\circ} \mathrm{C}$ à Wuhan). En janvier, à Vitim (Sibérie), l'écart à la normale a atteint la valeur exceptionnelle de $14,6^{\circ} \mathrm{C}$ ! Le 20 juin, Verkhoiansk (à $60^{\circ}$ de latitude) a enregistré la température extraordinaire de... $38^{\circ} \mathrm{C}$ (en hiver, le thermomètre y était descendu à $-57,2^{\circ} \mathrm{C}$ !). Les températures maximales de l'année ont souvent dépassé $50{ }^{\circ} \mathrm{C}$ au Moyen-Orient (53,5 ${ }^{\circ} \mathrm{C}$ à Mehran (Iran), 53,1 ${ }^{\circ} \mathrm{C}$ à Sulaibiya (Koweït), $53{ }^{\circ} \mathrm{C}$ à Amarah (Iran), 52, $5^{\circ} \mathrm{C}$ à Abadan (Iran), $52,1^{\circ} \mathrm{C}$ à Koweït City, $51,8^{\circ} \mathrm{C}$ à Bagdad (Irak), $51,1{ }^{\circ} \mathrm{C}$ à Rafha (Arabie saoudite), $51^{\circ} \mathrm{C}$ à Jacobabad (Pakistan), 50,2 ${ }^{\circ} \mathrm{C}$ à Bassora (Irak). En juillet, Bahrein a connu 23 jours consécutifs à plus de $40{ }^{\circ} \mathrm{C}$... Début septembre, au MoyenOrient, une vague de chaleur a fait monter les thermomètres à $48,9{ }^{\circ} \mathrm{C}$ à Eilat (record absolu en Israël), $48,5^{\circ} \mathrm{C}$ à Aqaba (Jordanie), $47,7{ }^{\circ} \mathrm{C}$ à Koweït City, 46,2 ${ }^{\circ} \mathrm{C}$ à Athalassa (Chypre), $44,6{ }^{\circ} \mathrm{C}$ à Damas (Syrie), $44,3{ }^{\circ} \mathrm{C}$ à Médine (Arabie saoudite), 43,7 ${ }^{\circ} \mathrm{C}$ à Bahrein et $42,3^{\circ} \mathrm{C}$ à Jérusalem (record absolu pour cette station).

En Afrique, sur les 85 stations analysées par le DWD, 83 ont eu une moyenne excédentaire. 2020 occupe le quatrième rang de la série de 111 ans de relevés. Les températures les plus élevées de l'année ont été enregistrées au Sahara, au Sahel et en Afrique du nord : $48,7^{\circ} \mathrm{C}$ à Ain Salah (Algérie), $48,1{ }^{\circ} \mathrm{C}$ à Bilma (Niger), $47,4^{\circ} \mathrm{C}$ à Touggourt (Algérie), $47,2^{\circ} \mathrm{C}$ à Marrakech (Maroc), $47{ }^{\circ} \mathrm{C}$ à El Goléa (Algérie), $46,4^{\circ} \mathrm{C}$ à Nouakchott (Mauritanie), $46,3{ }^{\circ} \mathrm{C}$ à Dori (Burkina Faso), $45,5^{\circ} \mathrm{C}$ à Agadez (Niger), $45^{\circ} \mathrm{C}$ à Niamey (Niger), Zinder (Niger) et Saint-Louis (Sénégal) et $44,3{ }^{\circ} \mathrm{C}$ à Médenine (Tunisie) le 3 octobre. Le record appartient à Gabès (Tunisie) où, sous un coup de sirocco, l'on a enregistré $50^{\circ} \mathrm{C}$ le 3 août après $48^{\circ} \mathrm{C}$ la veille. 
En Amérique du Nord, les 85 stations citées par le DWD ont toutes connu des températures annuelles supérieures aux normales. L'année 2020 est au dixième rang dans la série de 111 ans de relevés. Dès avril, la chaleur est présente à Cuba $\left(39,7^{\circ} \mathrm{C}\right.$ à Veguitas) et à Haïti ( $37^{\circ} \mathrm{C}$ à Port-au-Prince). Le 27 mai, Montréal enregistre $36,6{ }^{\circ} \mathrm{C}$, record pour mai. En août, les écarts les plus importants se localisent dans l'ouest $\left(+4,5{ }^{\circ} \mathrm{C}\right.$ à Phoenix, $+4,2{ }^{\circ} \mathrm{C}$ à Salt Lake City, $+4,1^{\circ} \mathrm{C}$ à Las Vegas). Le 16 août est une date historique : à Furnace Creek, dans la vallée de la Mort, on enregistre une température de $54,4{ }^{\circ} \mathrm{C}$, nouveau record mondial absolu ! Ailleurs, les maximums affichent $47,8{ }^{\circ} \mathrm{C}$ à Phoenix (Arizona), $45,6{ }^{\circ} \mathrm{C}$ à Las Vegas (Nevada), 43,9 ${ }^{\circ} \mathrm{C}$ à Tucson (Arizona), 43,3 ${ }^{\circ} \mathrm{C}$ à El Paso (Texas), $43,1^{\circ} \mathrm{C}$ à Merida (Mexique), $42,2^{\circ}$ à Abilene (Texas), $40{ }^{\circ} \mathrm{C}$ à Salt Lake City (Utah). La chaleur et la sécheresse ont favorisé de nombreux incendies dans l'ouest du continent.

En Amérique du Sud, sur 54 stations retenues par le DWD, 46 ont eu une moyenne excédentaire. L'Amérique du Sud est la région du globe où l'écart à la normale est le moins marqué ; quelques stations ont même un écart négatif ( $\mathrm{La} \mathrm{Paz}$, Cordoba). Le Paraguay connaît les températures les plus élevées $\left(44,4{ }^{\circ} \mathrm{C}\right.$ à Filadelfia le 4 octobre, $44^{\circ} \mathrm{C}$ à Mariscal Estigarr, $42,8^{\circ} \mathrm{C}$ à Asuncio, $43,6{ }^{\circ} \mathrm{C}$ à Concepcion), suivi par l'Argentine $\left(43,6^{\circ} \mathrm{C}\right.$ à Resistencia, $42,6{ }^{\circ} \mathrm{C}$ à Oran, $41^{\circ} \mathrm{C}$ à Catamarca)

En Océanie, 62 stations sur 63 ont eu une moyenne excédentaire, mais l'écart à la normale est peu important : $1,6^{\circ} \mathrm{C}$ à Hawaï et à Giles (Australie) et $1,1^{\circ} \mathrm{Cà}$ Nouméa(Nouvelle-Calédonie). En Australie, les températures maximales ont atteint $48,9^{\circ} \mathrm{C}$ le 4 janvier près de Sydney, $46,9^{\circ} \mathrm{C}$ à Smithville, $46,7^{\circ} \mathrm{C}$ à Cobar, $44,8^{\circ} \mathrm{C}$ à Kalgoorlie, $44{ }^{\circ} \mathrm{C}$ à Canberra et $43,7{ }^{\circ} \mathrm{C}$ à Alice Springs.

Comme les années précédentes, les régions arctiques ont été marquées par un réchauffement sensible. Les écarts aux normales annuelles affichent jusqu'à $6,7^{\circ} \mathrm{C}$ à l'île de Vize (mer de Kara), 6,5 ${ }^{\circ} \mathrm{C}$ à Khatanga (nord Sibérie), au cap Tscheljuskin (nord Sibérie) et à Dikson (mer de Kara), $3{ }^{\circ} \mathrm{C}$ à Uelen (détroit de

(a)

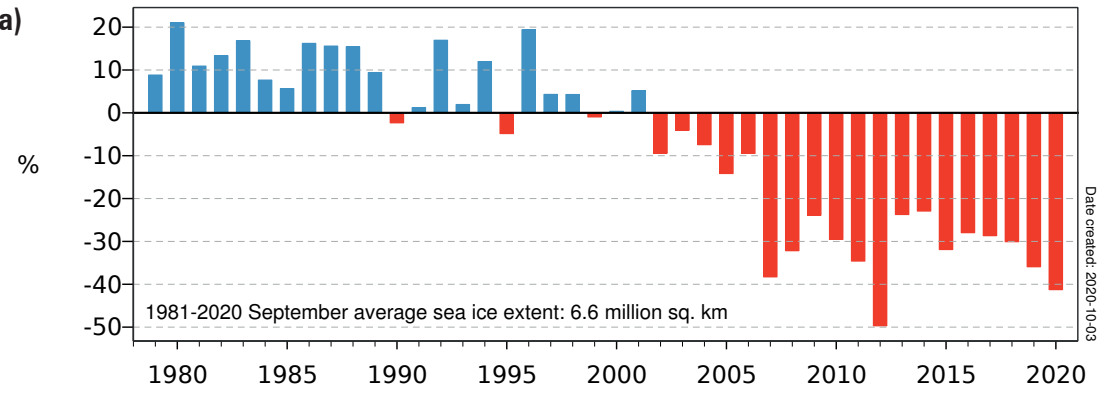

(b)

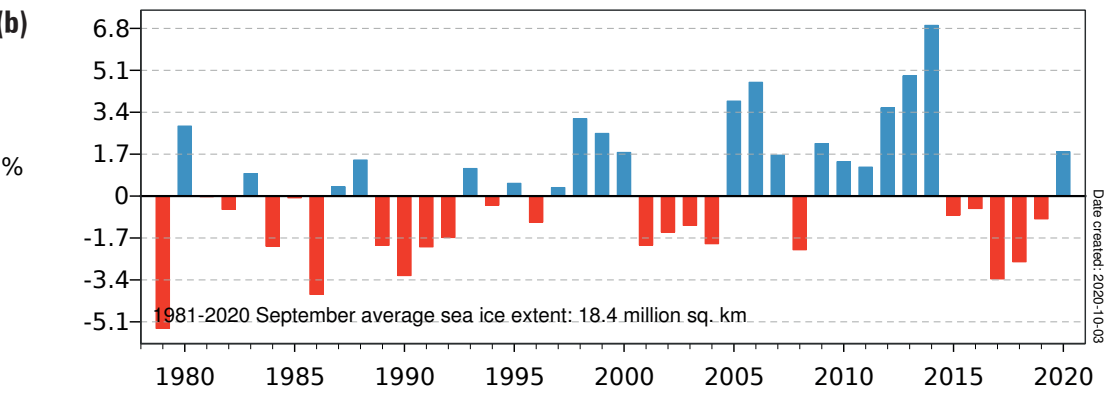

Figure 4. Évolution de la banquise (a) arctique (b) antarctique de 1979 à 2020 (anomalie en \% par rapport à la moyenne 1981-2010). Source : https://climate.copernicus.eu/sea-ice-cover-september-2020.

Béring) et à Svalbard (Spitzberg), $2,9^{\circ} \mathrm{C}$ à Eure-ka (Nunavut-Canada). En novembre, l'écart est exceptionnel : $16{ }^{\circ} \mathrm{C}$ à l'île de Vize, $13,7^{\circ} \mathrm{C}$ au cap Tscheljuskin, $13,3{ }^{\circ} \mathrm{C}$ à Khantaga, $9,5^{\circ} \mathrm{C}$ à Barrow (Alaska). Les températures maximales absolues affichent $21,7{ }^{\circ} \mathrm{C}$ à Svalbard, $21,9^{\circ} \mathrm{C}$ à Eureka, $27,3^{\circ} \mathrm{C}$ à Inuvik (Territoires du NordOuest, Canada) et $27,8^{\circ} \mathrm{C}$ à Fairbanks (Alaska). La banquise arctique ne cesse de reculer (figure 4a) : le 15 septembre, date de son extension minimale, elle s'étend sur 3,74 millions de kilomètres carrés (deuxième valeur la plus faible depuis 1979 ; moyenne 6,6 millions de kilomètres carrés). Durant l'été 2020, 331 navires ont emprunté la route maritime du nord (contre 277 en 2019), dont 62 de la mer de Barents au détroit de Béring (37 en 2019). Ce sont des records... En revanche, la superficie de la banquise antarctique a très légèrement augmenté (figure $4 b$ ).

L'activité cyclonique a été importante dans l'Atlantique Nord : 30 systèmes dont 17 tempêtes et 13 ouragans (la moyenne est de 6, mais 15 en 2005). À partir du 18 septembre, il a fallu, comme en 2005, utiliser l'alphabet grec pour nommer les ouragans... Les plus intenses ont été : Laura (du 20 au 29 août; vent de $240 \mathrm{~km} / \mathrm{h}$ ), Teddy (du 12 au 23 septembre), Delta (du 5 au 10 octobre, vent de $230 \mathrm{~km} / \mathrm{h}$, chute de $52 \mathrm{hPa}$ en 36 heures), Eta (du 31 octobre au 13 novembre, vent de $240 \mathrm{~km} / \mathrm{h}$ ) et Iota (du 13 au 18 novembre, en Amérique centrale, vent de $260 \mathrm{~km} / \mathrm{h}$, le plus meurtrier). Dans le nord-ouest du Pacifique, on a enregistré 23 systèmes cycloniques dont 11 tempêtes et 12 typhons (valeur inférieure à la moyenne) ; le plus intense a été Goni fin octobre sur les Philippines (rafales de $350 \mathrm{~km} / \mathrm{h}$ et pression minimale de $885 \mathrm{hPa}$ ). Le nord de l'océan Indien a vu cinq systèmes dont quatre cyclones; le plus important a été Amphan du 15 au 25 mai dans le golfe du Bengale (vent de $240 \mathrm{~km} / \mathrm{h}$; plus de 113 morts et 13,2 milliards de dollars de dégâts) ; Gati a affecté la Somalie et le Yémen en novembre. Dans le sudouest de l'océan Indien, dix systèmes cycloniques dont quatre tempêtes tropicales et six cyclones ont été signalés, le plus important étant Harold qui a touché l'est de Madagascar et l'île Maurice du 12 au 19 mars. Enfin, dans le sud-ouest du Pacifique, Yasa a dévasté les îles Fidji en décembre (rafale de $345 \mathrm{~km} / \mathrm{h}$, pression minimale de $899 \mathrm{hPa}$ ).

Guy Blanchet

Directeur honoraire du Centre de climatologie de l'université Claude Bernard-Lyon1 